Proceedings of SALT 31: 466-483, 2021

\title{
Challenge problems for a theory of degree multiplication (with partial answer key)*
}

\author{
Elizabeth Coppock \\ Boston University
}

\begin{abstract}
This paper offers a theory of degree multiplication in natural language semantics. Motivation for the development such a theory comes from proportional readings of quantity words and rate expressions such as miles per hour. After laying out a set of 'challenge problems' that any good theory of degree multiplication should be able to handle, I set about solving them, borrowing mathematical tools from quantity calculus. These algebraic foundations are integrated into a compositional Montagovian framework, yielding a system that can solve, or partially solve, some of the problems.
\end{abstract}

Keywords: degree multiplication, divisor-marking, ratios, proportional readings

\section{Motivation}

This paper makes the case that the foundations for degree semantics should furnish operations of multiplication and division among degrees, and shows how to achieve that, building on tools from quantity calculus. Let us begin by fleshing out what that means and seeing how it might be useful.

One motivation for having degree multiplication in the foundations for our theory of semantics comes from 'proportional' readings of quantity words like few and many (as opposed to 'cardinal' readings):

(1) Few egg-laying mammals suckle their young.

There are actually quite a large number of egg-laying mammals that suckle their young, in terms of raw numbers, but relative to the total number of egg-laying mammals, the proportion is low; that's what makes this a 'proportional reading' (Partee 1989).

* This work has benefitted from discussions with audience members at the NYU Philosophy of Language Workshop and the MIT Semantics Triangle, especially Lucas Champollion, Friederike Moltmann, Maša Močnik, Ying Gong, Hayley Ross, and Kai von Fintel. Thanks also to David Alvarez (BU undergraduate research assistant, summer 2019) for a first pass on the analysis of per. 
Challenge problems for a theory of degree multiplication

One recent approach to the analysis of these kinds of cases is to involves the idea that degrees can be proportions (Solt 2009, Bale \& Schwarz 2019). And one explicit manifestation of this idea is in Bale \& Schwarz's lexical entry for much/many:

$$
\lambda d \lambda P \lambda Q \cdot \mu(P \cap Q) \geq d
$$

where $\mu$ has a contextually set value, e.g. one of $\mu_{\text {weight }}, \mu_{\text {volume }}, \mu_{\text {length }}, \mu_{\#}$,

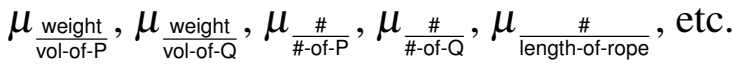

This lexical entry appeals to an under-specified measure function $\mu$, which can take on any of several more specific values, including the ones shown in the list they provide. Some of these $\mu$ 's measure objects along a dimension that is expressed as a fraction. What does it mean to measure something along a dimension that is expressed as a fraction? Is the value of such a measurement a degree that is itself a quotient of degrees? A theory of degree multiplication can answer such questions.

Another place where multiplication involving degrees shows up in the existing literature is in the analysis of percent, which has interesting conservativity-violating usages that have been the subject of some recent discussion (Ahn 2012; Sauerland 2014; Ahn \& Sauerland 2017; Sauerland \& Pasternak to appear): ${ }^{1}$

(3) The company hired 30 percent women.

Sauerland \& Pasternak (to appear) analyze percent as follows:

$$
\lambda D_{d t} \lambda n_{n} \lambda D_{d t}^{\prime} . D^{\prime} \subseteq D \wedge \max \left(D^{\prime}\right) \geq \frac{n}{100} \times \max (D)
$$

This lexical entry appeals to a multiplication operation between a number that is expressed as a fraction and a degree - the maximum in a given set of degrees.

Taking per out of percent exposes a wider range of cases where the idea of quotient is intuitively expressed in natural language: parts per million, miles per hour, dollars per couple, hospitals per capita. And it's not just per; there there are other words and constructions that express the same idea: situps a day, cents on the dollar, and cents for every dollar. Along with division, there are also words and constructions that express multiplication, such as twice as tall (which presumably involves multiplication of a number times a degree), cubic centimeters (centimeters times centimeters), and arguably 3 apples at \$2 per apple (which is \$6).

There are two previous approaches to the semantics of per that have been offered. Panaitescu \& Tovena (2019) offer an analysis of Romanian de and Italian per inspired by Rothstein (1995), treating the phenomenon as a kind of distributivity involving matching between two domains. ${ }^{2}$ This approach does not make use of the concept of

1 See also Ahn \& Sauerland 2015; Li 2018; Solt 2018; Spathas 2019; Pasternak 2019; Coppock under review, and other contributions to a forthcoming special issue of Glossa on the topic.

2 I learned of this work only after the copy-editing stage, so I must delay a comparison between their 
division, and relies instead solely on familiar quantificational mechanisms. Rawlins (2013), who uses per to eludicidate the formal semantics of 'adverbs of space and time' like quickly and slowly (Cresswell 1977b), suggests the kind of divisionbased approach that I pursue here. As Rawlins (2013) discusses, measure phrases constructed with per can serve as differential arguments of comparative adverbs, but only when the modified verb phrase has the right aktionsart. The notion of degree division appears again in Rawlins's analysis: Building on Alrenga's (2007) notion of dimension as a set of degrees, Rawlins defines the 'ratio dimension' of Dimension A and Dimension B as the set of degrees obtained by dividing a degree in Dimension A by a degree in Dimension B, "settting aside some issues in properly defining division" (Rawlins 2013: 184).

Within formal semantics, foundations for degree multiplication have not yet been laid down. Cresswell (1977a), who provided the first serious foundations for degree semantics, only provided a comparison operation. Klein (1991) contributed addition, through concatenation. Sassoon (2010) and van Rooij (2011) explicitly discuss multiplication within a particular dimension, building on measurement theory (Krantz, Luce, Suppes \& Tversky 1971). Thanks to their work, we can analyze cases like twice as tall. But if we want to multiply and divide across dimensions, as the examples with per seem to require, then we will need more foundational changes to our theory of semantics.

To nurture and guide the development of a theory of degree multiplication, I hereby propose a set of "challenge problems". 3 These are inferences whose validity we should be able to capture if we have a good theory of degree multiplication.

Sainetra walked at $5 \mathrm{mph}$ for 3 hours.

(Challenge Problem 1) Therefore, Sainetra walked 15 miles.

(6) Zahra did 30 situps a day for a week.

(Challenge Problem 2) Therefore, Zahra did 210 situps in one week.

(7) Tickets cost $\$ 5$ per couple. Therefore, tickets for 3 couples costs $\$ 15$.

(Challenge Problem 3)

To preview where we will end up, kilometers per hour will be analyzed as a quotient that can be expressed as a fraction:

$$
\text { kilometers per hour } \rightsquigarrow \frac{\mathrm{km}}{\text { hour }}
$$

Here, the numerator is a particular quantity corresponding to kilometer, and the approach and mine to future work.

3 I am inspired here by the "Recognizing Textual Entailment" challenge in natural language processing (Dagan, Glickman \& Magnini 2006). 
Challenge problems for a theory of degree multiplication

denominator is a particular quantity, the quantity corresponding to hour. This is not your grandmother's division. It's not division among numbers, but rather among degrees. We can't take that for granted; we have to spell out what that means. Luckily, there is an 200-year intellectual tradition devoted to doing exactly that: the study of quantity calculus.

\section{Quantity calculus}

Quantity calculus is the mathematical study of quantities. In this context, a 'quantity' is defined as "a property of a phenomenon, body or substance, where the property has a magnitude that can be expressed as a number and a reference" (JCGM 2012). The 'reference' here could be a unit like 'kilometer'. So the magnitude of a particular quantity could be, for example, 'five kilometers'. Examples include the radius (of a particular circle), and the wavelength of a particular sample of radiation. ${ }^{4}$

In the literature on quantity calculus, it is generally taken for granted that there are three operations to characterize:

- product of quantities

- product of a number times a quantity

- addition of quantities of the same kind

So any two quantities can be multiplied together, but addition is more restricted.

The history of this endeavor goes back almost exactly two centuries; see de Boer 1994 for a helpful overview of the intellectual history. Yet the algebraic foundations for quantity calculus remain a topic of discussion to this day. I will be drawing on a recent proposal by Raposo $(2018,2019)$, who distinguishes between two different approaches to the algebraic foundations for quantity calculus: a unit-centric approach, where a quantity is defined as a combination of a number and a unit, and a dimension-centric approach, under which "the dimension is an intrinsic property of a quantity, in contrast to its numerical value, which depends on the unit chosen, or the unit itself, which can be changed arbitrarily." So, for example, the quantity named by 'five kilometers' bears no intrinsic relation to the number five, but it does bear an intrinsic relation to the dimension 'length'.

4 JCGM (2012) distinguish between specific 1 quantities like the radius of a particular circle and general quantities like 'radius' in general. The specific quantities could be thought of as similar to tropes insofar as they inhere in particular objects, but I see them as sharable across objects, so for example if you and I are the same height then the quantity corresponding to my height would be the same as the quantity corresponding to your height. So the specific quantities are like trope types. I will use 'quantity' in the specific sense here, and I take it to be synonymous with 'degree'. 
I will adopt a dimension-centric approach. We will start, therefore, with a set of basic dimensions, written $\mathscr{B}$. We could, for example, start with the seven basic dimensions identified in the International System of Units (SI). Each of these has a designated symbol and a corresponding base unit:

$\begin{array}{ll}\text { Dimension } & \text { Base unit } \\ \mathrm{L}-\text { length } & \text { meter }(\mathrm{m}) \\ \mathrm{M}-\text { mass } & \text { kilogram }(\mathrm{kg}) \\ \mathrm{T}-\text { time } & \text { second }(\mathrm{s}) \\ \mathrm{I}-\text { electric current } & \text { ampere }(\mathrm{A}) \\ \Theta-\text { thermodynamic temperature } & \text { kelvin }(\mathrm{K}) \\ \mathrm{N}-\text { amount of substance } & \text { mole }(\mathrm{mol}) \\ \mathrm{J}-\text { luminous intensity } & \text { candela }(\mathrm{cd})\end{array}$

Now, a particular quantity could have as its dimension a product of basic dimensions. For example, if $h$ is Plank's constant, ${ }^{5}$ then the dimension of $h$ is mass times length squared, divided by time:

$$
\operatorname{dim}(h)=\mathrm{M} \cdot \mathrm{L}^{2} \cdot \mathrm{T}^{-1}
$$

The basic dimensions can be multiplied together to get derived dimensions. They can also be multiplied by themselves - length times length, for example.

The set of dimensions $\mathscr{D}$ forms a group under product, which means:

- if $\mathrm{A}, \mathrm{B} \in \mathscr{D}$, then $\mathrm{A} \cdot \mathrm{B} \in \mathscr{D}$

- $\mathscr{D}$ has an identity element $\mathbf{1}_{\mathscr{D}}$, such that for every $\mathrm{D} \in \mathscr{D}: \mathrm{D} \cdot \mathbf{1}_{\mathscr{D}}=\mathbf{1}_{\mathscr{D}} \cdot \mathrm{D}=\mathrm{D}$

- There is an inverse $D^{-1}$ for every $D \in \mathscr{D}$ : an element such that $\mathrm{D} \cdot \mathrm{D}^{-1}=\mathbf{1}_{\mathscr{D}}$

These are the properties that define a group: closure under product, the existence of a multiplicative identity element, and the existence of multiplicative inverses for each member of the set.

There do exist quantities that have as their dimension this multiplicative identity element $\mathbf{1}_{\mathscr{D}}$. These are sometimes called 'dimensionless quantities', although the term is a bit of a misnomer. Examples include ratios of two quantities of the same kind. 'Relative permeability' is an example from physics; 'dollars earned per dollars saved' is a more accessible example, with dollars as the unit on both the numerator and the denominator. Numbers of entities, like number of molecules in a given sample, are also considered 'dimensionless'. We will come back to this fact.

A dimension can be raised to any integer power. For a given dimension $D$,

5 Apparently the Planck constant multiplied by a photon's frequency is equal to a photon's energy. 
Challenge problems for a theory of degree multiplication

$$
\begin{array}{ll}
\mathrm{D}^{0}=\mathbf{1}_{\mathscr{D}} & \\
\mathrm{D}^{1}=\mathrm{D} & \mathrm{D}^{-1}: \text { multiplicative inverse of } \mathrm{D} \\
\mathrm{D}^{2}=\mathrm{D} \cdot \mathrm{D} & \mathrm{D}^{-2}=\left(\mathrm{D}^{-1}\right)^{2} \\
\mathrm{D}^{3}=\mathrm{D} \cdot \mathrm{D}^{2} & \mathrm{D}^{-3}=\left(\mathrm{D}^{-1}\right)^{3} \\
\text {...and so on: } \mathrm{D}^{k}=\mathrm{D} \cdot \mathrm{D}^{k-1} & \ldots \text { and so on: } \mathrm{D}^{-k}=\left(\mathrm{D}^{-1}\right)^{k}
\end{array}
$$

Now, each dimension in $\mathscr{D}$ has a unique expression as the product of basic dimensions raised to integer powers. Thus, if our set of basic dimensions $\mathscr{B}$ is the set of basic SI dimensions, then each dimension in $\mathscr{D}$ can be written as follows:

$$
\mathrm{D}=\mathrm{L}^{n_{1}} \cdot \mathrm{M}^{n_{2}} \cdot \mathrm{T}^{n_{3}} \cdot \mathrm{I}^{n_{4}} \cdot \Theta^{n_{5}} \cdot \mathrm{N}^{n_{6}} \cdot \mathrm{J}^{n_{7}}
$$

where $n_{1}, \ldots, n_{7}$ are integers.

Now let us bring quantities into the picture. The set of quantities is written $\mathscr{Q}$, and each quantity $q \in \mathscr{Q}$ is mapped by a function dim to its unique dimension.

$$
\mathscr{Q} \stackrel{\operatorname{dim}}{\longrightarrow} \mathscr{D}
$$

For example, quantities of mass will be mapped by dim to the dimension mass: If $3 \mathrm{~kg}$ names a particular mass, then $\operatorname{dim}(3 \mathrm{~kg})=\mathrm{M}$. The inverse of the dimension mapping, written $\mathrm{dim}^{-1}$, identifies all of the quantities sharing a given dimension. Thus, $\operatorname{dim}^{-1}(M)$ denotes the set of quantities of mass. Rather than $\operatorname{dim}^{-1}$ (D) I will write $\mathscr{Q}_{\mathrm{D}}$ to denote the set of quantities with dimension D. Note that, as a consequence, Alrenga's (2007) conception of dimensions as sets of degrees/quantities can straightforwardly be reconstrcted because dimensions (which are primitives here) determine a set of quantities.

On Raposo's (2019) system, the space of quantities is organized as a fiber bundle. Each dimension corresponds to a fiber within the bundle. Within a fiber it is possible to add and multiply but across fibers it is only possible to multiply. Each one of these fibers is a vector space over the real numbers: For all dimensions D, the set of quantities with dimension $\mathrm{D}$, written $\mathscr{Q}_{\mathrm{D}}$, is a vector space over the real numbers (written $\mathbb{R}$ ), so:

- There exists a zero element $\mathbf{0}_{\mathrm{D}} \in \mathscr{Q}_{\mathrm{D}}$ such that for any $q \in \mathscr{Q}_{\mathrm{D}}$ :

$$
q+\mathbf{0}_{\mathbf{D}}=q
$$

- For any $q \in \mathscr{Q}_{\mathrm{D}}$, there is an additive inverse element $-q \in \mathscr{Q}_{\mathrm{D}}$ such that:

$$
q+(-q)=\mathbf{0}_{\mathbf{D}}
$$


- There is a multiplicative identity element 1 from $\mathbb{R}$ s.t. for any $q \in \mathscr{Q}_{\mathrm{D}}$ :

$$
q * \mathbf{1}=\mathbf{1} * q=q
$$

Note that the zero element $\mathbf{0}_{\mathbf{D}}$, which can also be called an 'additive identity element', is specific to the dimension; there is one zero element per dimension. Note also that the multiplicative identity is just the real number 1 .

Since for all dimensions $\mathrm{D}, \mathscr{Q}_{\mathrm{D}}$ is a vector space over $\mathbb{R}$, it follows that for any $q, q_{1}, q_{2}, q_{3} \in \mathscr{Q}$ and scalars $\alpha, \alpha_{1}, \alpha_{2} \in \mathbb{R}$ :

- $q_{1}+q_{2} \in \mathscr{Q}_{\mathrm{D}}$

- $\alpha * q \in \mathscr{Q}_{\mathrm{D}}$

- $q_{1}+q_{2}=q_{2}+q_{1}$

- $q_{1}+\left(q_{2}+q_{3}\right)=\left(q_{1}+q_{2}\right)+q_{3}$

- $\alpha_{1} *\left(\alpha_{2} * q\right)=\left(\alpha_{1} \times \alpha_{2}\right) * q$

- $\alpha *\left(q_{1}+q_{2}\right)=\alpha * q_{1}+\alpha * q_{2}$

- $\left(\alpha_{1}+\alpha_{2}\right) * q=\alpha_{1} * q+\alpha_{2} * q$ (closure under addition)

(closure under scalar multiplication)

(commutativity of + )

(associativity of + )

(compatibility of $*$ and $\times$ )

(distributivity 1)

(distributivity 2)

Basically: addition and multiplication are well-behaved. One thing to note here is that real number multiplication is distinguished in the notation from the kind of multiplication that involves quantities ( $\times$ vs. $*$, respectively; $\mathrm{cf}$. also $\cdot$ ).

Now that we have the fibers in place, the next step is to bundle them together using the product operation. Because not every quantity has a multiplicative inverse, the space of quantities does not form a group under the product operation; $\langle\mathscr{Q}, *\rangle$ is, rather, an abelian monoid (called 'abelian' because * is commutative):

- If $q_{1}, q_{2} \in \mathscr{Q}$, then $q_{1} * q_{2} \in \mathscr{Q}$

- There is a multiplicative identity element $\mathbf{1}$ such that for all $q \in \mathscr{Q}$ :

$$
q * \mathbf{1}=\mathbf{1} * q=q
$$

- If $q_{1}, q_{2}, q_{3} \in \mathscr{Q}$ then $q_{1} *\left(q_{2} * q_{3}\right)=\left(q_{1} * q_{2}\right) * q_{3}$

(associativity)

- $q_{1} * q_{2}=q_{2} * q_{1}$

(commutativity)

The most important part is the fact that the set of quantities is closed under product; this is what binds the fibers together into a bundle. 
Challenge problems for a theory of degree multiplication

Again, not every quantity has an inverse. In particular, none of the zeros $\mathbf{0}_{\mathrm{D}}$ (for $\mathrm{D} \in \mathscr{D}$ ) has a multiplicative inverse. In other words, you can't divide by (any of the) zero(es). But for every non-zero quantity $q \in \mathscr{Q}$, there is an inverse $q^{-1}$ :

$$
q * q^{-1}=\mathbf{1}
$$

In other words, the set of non-zero quantities forms a group under multiplication. This comes in handy, because it gives us confidence that we can divide by a given quantity as long as we know that it is not a zero.

Recall that there is a mapping from the set of quantities to the set of dimensions called dim. There is also a mapping in the other direction called unit. For each dimension, there is a designated quantity in $\mathscr{Q}$ that serves as the unit quantity for that dimension. There are restrictions on the unit mapping: A unit quantity cannot be a zero, and unit must be a group homomorphism:

$$
\operatorname{unit}(\mathrm{A} \cdot \mathrm{B})=\operatorname{unit}(\mathrm{A}) * \operatorname{unit}(\mathrm{B})
$$

Gloss: "The unit of a product of dimensions is the product of units of the dimensions." Because a unit is non-zero quantity, we can divide by any unit (or any product of a unit with a non-zero scalar).

This concludes the presentation of Raposo's system. Now we have the tools necessary to represent kilometers per hour. If kilometer is the unit of length and hour is a non-zero number times the unit of time (e.g. hour $=60 *$ second), then we can represent kilometers per hour as kilometer times the multiplicative inverse of hour:

$$
\mathrm{km} * \text { hour }^{-1}
$$

We know that hour has a multiplicative inverse because it is a non-zero quantity.

\section{Representation language}

We are finally ready to start doing semantics. Let us now import the mathematical foundations we established in the previous section into a Montagovian framework. I define a formal representation language $\mathscr{L}_{\mathscr{Q}}$, which is a $\lambda$-categorial language, complete with lambda operators and universal and existential quantifiers, one that also has quantity calculus built into it. An expression in $\mathscr{L}_{\mathscr{Q}}$ is assigned a denotation relative to a model that incorporates the operations over degrees and dimensions that we have been discussing. More specifically, the semantic value of an expression $\phi$ in $\mathscr{L}_{\mathscr{Q}}$ is given by $\llbracket \phi \rrbracket^{M}$, where:

$$
M=\left\langle\mathscr{A}, \mathscr{V},\left\langle\mathscr{D}_{\mathscr{B}}, \cdot\right\rangle,\langle\mathscr{Q}, *,+\rangle, \text { unit, } \operatorname{dim}, I\right\rangle
$$


Here $\mathscr{A}$ is a set of individuals and $\mathscr{V}$ a set of events. As usual, I maps each constant of type $\tau$ to an element of $D_{\tau}$; this is familiar from traditional Montagovian frameworks. The other components are as described above:

- $\left\langle\mathscr{D}_{\mathscr{B}}, \cdot\right\rangle$ is an abelian group with basis $\mathscr{B}$, a finite set of dimensions

- $\operatorname{dim}$ is a surjection map from $\mathscr{Q}$ onto $\mathscr{D}_{\mathscr{B}}$

- Each $\operatorname{dim}^{-1}(\mathrm{D})=\mathscr{Q}_{\mathrm{D}}$ yields a one-dimensional vector space over $\mathbb{R}$

- $\langle\mathscr{Q}, *\rangle$ is an abelian monoid

- unit is a group homomorphism from $\mathscr{D}_{\mathscr{B}}$ to $\mathscr{Q}$

Each expression in $\mathscr{L}_{\mathscr{Q}}$ has a type, and a denotation in the corresponding domain; if $\alpha$ has type $\tau$ then $I(\alpha) \in D_{\tau}$. Most of the types are familiar. For type $t: D_{t}=\{0,1\}$. For type $e: D_{e}=\mathscr{A}$. For type $v: D_{v}=\mathscr{V}$. For type $n: D_{n}=\mathbb{R}$. The only slight difference is that expressions of type $d$ (for 'degree') are interpreted as members of the set of quantities: $D_{d}=\mathscr{Q}$. Complex types are defined as usual: If $\sigma$ and $\tau$ are types, then $\langle\sigma, \tau\rangle$ is a type, the type of functions from $D_{\sigma}$ to $D_{\tau}$.

To complete the specification of the representation language, we must import the multiplication and addition operations into it from the meta-language:

- $\llbracket \alpha+\beta \rrbracket^{M}=\llbracket \alpha \rrbracket^{M}+\llbracket \beta \rrbracket^{M}$

- $\llbracket \alpha \cdot \beta \rrbracket^{M}=\llbracket \alpha \rrbracket^{M} \cdot \llbracket \beta \rrbracket^{M}$

- $\llbracket \alpha * \beta \rrbracket^{M}=\llbracket \alpha \rrbracket^{M} * \llbracket \beta \rrbracket^{M}$

- $\llbracket \alpha^{n} \rrbracket^{M}=\left(\llbracket \alpha \rrbracket^{M}\right)^{n}$

We will also make use of the following abbreviation, use fractions to signify division:

$$
\alpha * \beta^{-1} \equiv \frac{\alpha}{\beta}
$$

Multiplying by the multiplicative inverse is the same as dividing.

Here are denotations for some constants of type $d$ in the representation language: $\llbracket \mathrm{m} \rrbracket^{M}=\mathrm{m}=$ unit(L). $\llbracket \mathrm{km} \rrbracket^{M}=1000 * \mathrm{~m} . \llbracket \mathrm{s} \rrbracket^{M}=\mathrm{s}=$ unit( $\left.\mathrm{T}\right) . \llbracket$ minute $\rrbracket^{M}=60 * \mathrm{~s}$. $\llbracket$ hour $\rrbracket^{M}=60 * 60 * \mathrm{~s}$. Again, constants of type $d$ are interpreted as quantities.

Expressions of English will be translated to $\mathscr{L}_{\mathscr{Q}}$ by a function $\rightsquigarrow$. For example, the word meter will be translated as $\mathrm{m}$. Crucially, we can now give a lexical entry for per:

$$
\operatorname{per} \rightsquigarrow \lambda d \lambda q \cdot q * d^{-1}
$$




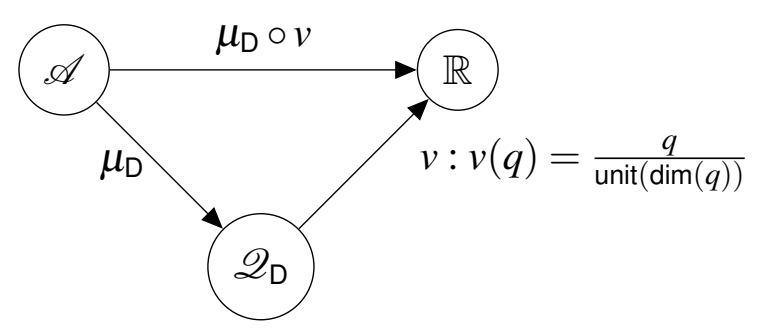

Figure 1 The Lønning Triangle

On this treatment, per takes two degrees ( $d$ for 'degree' or 'denominator' and $q$ for 'quantity'), and gives back $q$ times the multiplicative inverse of $d$, or $\frac{q}{d}$.

Now, using the composition rule of Function Application (Heim \& Kratzer 1998) we can compositionally derive an appropriate meaning for kilometers per hour as follows:

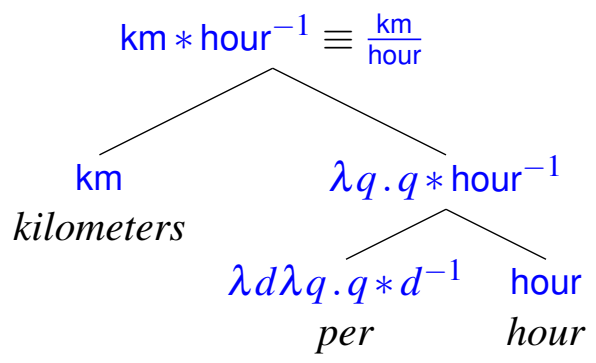

We have our first compositional analysis of a fractional quantity!

To solve the challenge problems, we will need to link individuals and events to quantities. I will adopt a version of what might be called the Lønning Triangle (Lonning 1987), shown in Figure 1. It breaks up the relation between individuals and numbers into two steps: assigning an individual a quantity, and mapping from quantities to numbers. On Champollion's (2017) theory, unit terms like kilo denote the latter kinds of functions, from quantities to numbers. The proposal here is similar, insofar as the denotation of unit terms will be interdefinable with such functions.

On the left edge of the triangle, I assume that, for every dimension D, there is a function $\mu_{\mathrm{D}}$ (as a first pass). ${ }^{6}$ For the right edge of the triangle, it would be possible to define a function from quantities to numbers. This would be a valuation function $v$ which assigns to a quantity $q$ the quotient of $q$ and the unit for $\operatorname{dim}(q)$. For example,

$6 \mathrm{~A}$ measure function $\mu_{\mathrm{D}}$ could be viewed in a number of ways. If quantities are viewed as kinds (Anderson \& Morzycki 2015; Scontras 2017), then it could be an instantiation relation; if quantities are viewed in terms of tropes (Moltmann 2009), $\mu_{\mathrm{D}}(x)$ could be seen as mapping $x$ to a particular trope type that $x$ bears an instance of. Both of these are consistent with the proposal here. 
supposing that $\mathrm{km}$ is the unit of length:

$$
v(3 \mathrm{~km})=\frac{3 \mathrm{~km}}{\operatorname{unit}(\operatorname{dim}(3 \mathrm{~km}))}=\frac{3 \mathrm{~km}}{\operatorname{unit}(\mathrm{L})}=\frac{3 \mathrm{~km}}{\mathrm{~km}}=3
$$

I happen to be treating units as particular quantities, but we can easily go back and forth between these two ways of viewing the semantics of unit terms.

Notice that we already have a handle on the notation from Bale \& Schwarz (2019) where fractions occur in the subscript of the $\mu$ functions:

$$
\mu_{\frac{\text { weight }}{\text { vol-of-P }}}, \mu_{\frac{\text { weight }}{\text { vol-ot-Q }}}, \mu_{\frac{\#}{\#-\text { of-P }},}^{\#}, \mu_{\text {\#-of-Q }}^{\#}, \mu_{\frac{\#}{\text { length-of-rope }}}^{\#}
$$

We might not have everything in this list, but we have some meat to put on the bones of these kinds of representations. For example, $\mu_{\underline{\underline{\mathrm{T}}}}$ has a clear meaning.

\section{Challenge Problems}

We are now ready to approach the challenge problems. Let us begin with the first one, the most straightforward one:

Sainetra walked at $5 \mathrm{~km}$ per hour for 3 hours.

Therefore, Sainetra walked $15 \mathrm{~km}$.

To solve this problem, we need a principle like 'speed times time equals distance'.

$$
\operatorname{speed}(e) * \tau(e)=\sigma(e)
$$

Here, $\tau$ signifies the temporal trace function and $\sigma$ the spatial extent function (Krifka 1989; Champollion 2017). But let us write things a bit differently: $\mu_{\mathrm{T}}$ instead of $\tau$; $\mu_{\llcorner}$instead of $\sigma$; and $\mu_{\overline{\mathrm{T}}}$ instead of speed. Now the 'speed times time equals distance' principle can be derived from a deeper principle, one I am calling the $\mu$-product postulate (since it is a meaning postulate, in Montague's sense).

$$
\begin{aligned}
& \mu \text {-product postulate } \\
& \mu_{A}(x) * \mu_{B}(x)=\mu_{A \cdot B}(x)
\end{aligned}
$$

Dividing both sides by $\mathrm{B}$, we obtain the following corollary:

$$
\mu_{B}(x) * \mu_{B}(x)=\mu_{A}(x)
$$

In particular:

$$
\mu_{\underline{\mathrm{T}}}(x) * \mu_{\mathrm{T}}(x)=\mu_{\mathrm{L}}(x)
$$


Challenge problems for a theory of degree multiplication

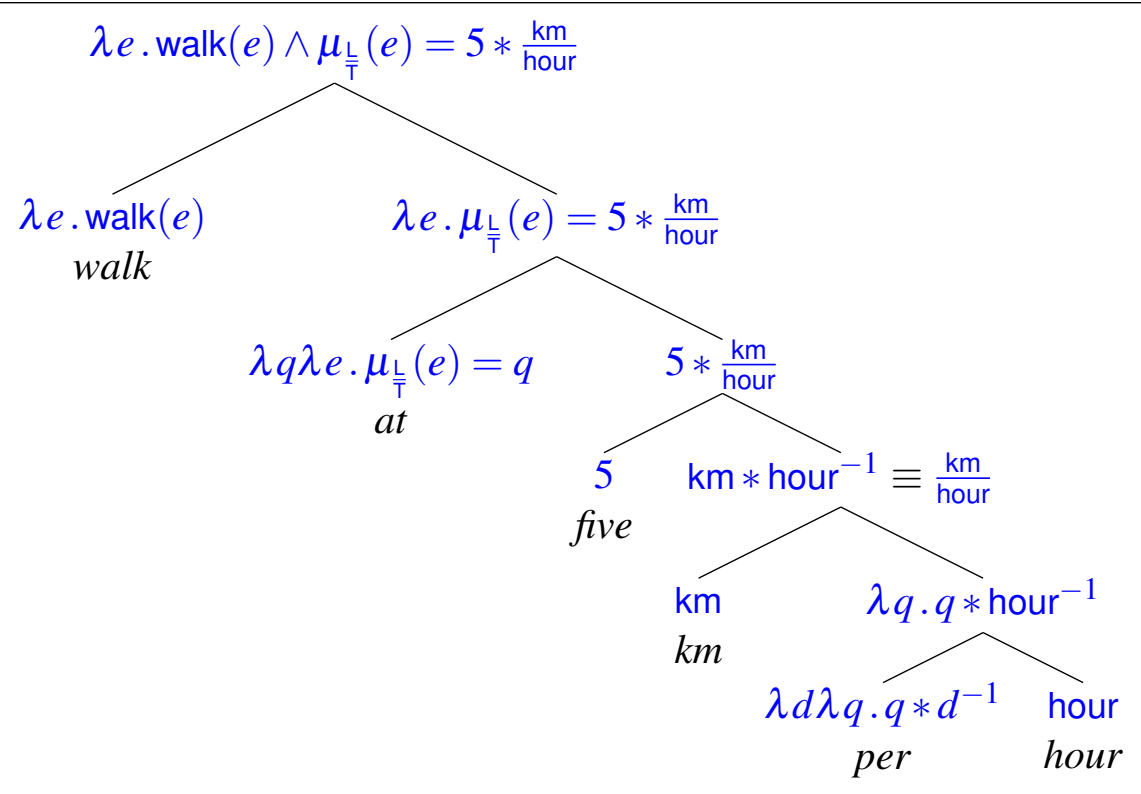

Figure 2 Derivation for walk at five $\mathrm{km}$ per hour.

In other words: speed times time equals distance.

We will also need a compositional analysis of walk at $5 \mathrm{~km}$ per hour. We already have a compositional analysis of $\mathrm{km}$ per hour. To combine it with five, I propose we use a composition rule that introduces multiplication (following loosely in the footsteps of Ionin \& Matushansky 2006). Let us assume at introduces a speed modifier of an event, yielding a property of an event (cf. the analysis of adverbs like quickly by Tenny 2000; Ernst 2004: i.a.). Walk also denotes a property of an event. These two properties combine via predicate modification, yielding a property that holds of a walking event whose speed is $5 \mathrm{~km}$ per hour.

Making the simplifying assumption that for three hours does nothing more than specify the duration of an event (and ignoring the fact that it contributes distributivity; cf. Champollion 2015), the VP in the premise of (11) denotes the following:

walk at $5 \mathrm{~km}$ per hour for three hours

$\rightsquigarrow \lambda e \cdot \operatorname{Walk}(e) \wedge \mu_{\frac{\mathrm{L}}{\mathrm{T}}}(e)=5 * \frac{\mathrm{km}}{\text { hour }} \wedge \mu_{\mathrm{T}}(e)=3 *$ hour

By the $\mu$-product postulate, any event that satisfies this description also satisfies the denotation of walk $15 \mathrm{~km}$.

$$
\text { walk } 15 \mathrm{~km} \rightsquigarrow \lambda e \cdot \operatorname{Walk}(e) \wedge \mu_{\mathrm{L}}(e)=15 * \mathrm{~km}
$$

Since the VP in the premise is a subset of the VP in the conclusion, and the two 
sentences have the same subjects and tense/aspect features, the premise entails the conclusion. Challenge problem solved!

Let us now move on to the second challenge problem, repeated just below, which will raise some interesting issues.

Zahra did 30 situps a day for a week.

Therefore, Zahra did 210 situps in one week.

Rather than a unit of measurement along a physical dimension, the numerator names a category of events: situps. One approach to this type of case would be to assume that for every property $P$, there is a different dimension \#P. For example, if $x$ is a collection of three bears, then you could say that $x$ measures three times the unit for the 'number of bears' dimension, along that dimension. (The resulting $\mu$ function would be much like Krifka's (1995) 'object unit' function.)

$$
\mu_{\text {\#bear }}(x)=3 * \text { unit(\#bear) ' } x \text { is three bears' }
$$

Similarly, an event of 30 situps might be said to measure 30 times the unit for the 'number of situps' dimension, along that dimension:

$$
\mu_{\# \operatorname{situp}}(e)=30 * \text { unit(\#situp) } \quad \text { ' } e \text { is } 30 \text { situps' }
$$

Let us adopt this assumption for now; we'll consider another alternative later.

Now for day. If we take it to mean 'mean solar day' then we can define it as a number of seconds: ${ }^{7}$

$$
\text { day } \equiv 24 *(60 *(60 * s))
$$

With these assumptions in place, we can treat 30 situps a day as 30 times the unit for the 'number of situps' dimension, divided by day:

$$
30 \text { situps a day } \rightsquigarrow 30 * \frac{\text { unit(\#situps) }}{\text { day }}
$$

The verb do seems to function as a light verb here, so we can treat do 30 situps a day as a property of events that measures that rate of situps per day.

$$
\text { do } 30 \text { situps a day } \rightsquigarrow \lambda e \cdot \frac{\mu_{\text {\#situps }}}{\mathrm{T}}(e)=30 * \frac{\text { unit(\#situps) }}{\text { day }}
$$

Adding for a week (again simplifying a bit, and treating it as a 7-day duration) yields:

7 A solar day is the time it takes for the sun to return to the same point in the sky; a sidereal day is the time it takes for the earth to return to the same point relative to the stars. Solar days vary in length throughout the year, but the average (mean) is 24 hours, whereas sidereal days are about four minutes shorter. It is also worth noting that we are treating 'day' as a quantity, or duration, rather than an object; see both Fillmore 1997 and Champollion 2016a,b for discussion of this distinction. 
Challenge problems for a theory of degree multiplication

$$
\begin{aligned}
& \text { do } 30 \text { situps a day for a week } \\
& \rightsquigarrow \lambda e \cdot \frac{\mu_{\text {\#situps }}}{\mathrm{T}}(e)=30 * \frac{\text { unit(\#situps) }}{\text { day }} \wedge \mu_{\mathrm{\top}}(e)=7 * \text { day }
\end{aligned}
$$

The $\mu$-product postulate, instantiated with the dimensions in question is as follows:

$$
\mu_{\frac{\# \text { situps }}{\mathrm{T}}}(x) * \mu_{\mathrm{T}}(x)=\mu_{\# \text { \#itups }}(x)
$$

Given this, any event that satisfies the description in (24) also satisfies the description do 210 setups in a week, assuming that in a week just specifies a 7-day duration:

$$
\begin{aligned}
& \text { do } 210 \text { situps in one week } \\
& \left.\rightsquigarrow \lambda e \cdot \mu_{\text {\#situps }}(e)=210 * \text { unit(\#situps }\right) \wedge \mu_{\top}(e)=7 * \text { day }
\end{aligned}
$$

Solved! Assuming that we can make that assumption that for every property $P$ there's a corresponding dimension 'number of $P$ '...

Let us briefly consider Challenge Problem 3 before returning to that assumption.

Tickets cost $\$ 5$ per couple.

Therefore, tickets for 3 couples costs $\$ 15$.

In this case, the denominator seems to name a unit on a 'number of entities' dimension. Supposing we represent the denominator as unit(\#couple), and introduce a dimension for money in dollars called $\$ \$$ whose unit is $\$$, then the meaning of $\$ 5$ per couple could be represented as:

$$
\frac{5 * \$}{\text { unit(\#couple) }}
$$

Then the premise and the conclusion, respectively, can be represented as follows (simplifying the generic to a universal, and finessing the plural on tickets):

$$
\begin{aligned}
& \text { Tickets cost } \$ 5 \text { per couple. } \\
& \rightsquigarrow \forall x\left[\text { tickets }(x) \rightarrow \mu_{\text {\#couple }}^{\$ \$}(x)=\frac{5 * \$}{\text { unit(\#couple })}\right]
\end{aligned}
$$

Tickets for 3 couples cost $\$ 15$.

$$
\left.\rightsquigarrow \forall x\left[\text { tickets }(x) \wedge \mu_{\text {\#couple }}(x)=3 * \text { unit }(\text { \#couple })\right] \rightarrow \mu_{\$ \$}(x)=15 * \$\right]
$$

The conclusion follows from the premise given the $\mu$-product postulate. But notice that there is something a bit strange about this treament. Tickets for three couples in the conclusion is treated as 'tickets with a value of 3 on the \#couple dimension'. How is it possible to measure tickets on the \#couple dimension? If the measure of $x$ along that dimension is the number of couples comprising $x$, then a bunch of tickets should not be able to measure three on this dimension; it should measure zero if its measure along this dimension is even defined. All this is to make it evident that the 
dimension in question is not the number of couples comprising $x$; it's the number of couples that $x$ is for, or the number of couples possessing $x$.

Suppose we introduce the following shorthand:

$$
\mu_{\text {\#couple }_{\text {Poss }}}(x)=3 * \text { unit }(\# \text { couple } \text { Poss }) \equiv|y: \operatorname{couple}(y) \wedge \operatorname{Poss}(y, x)|=3
$$

Then we can revise the representation of the premise and the conclusion as follows:

$$
\begin{aligned}
& \text { Tickets cost } \$ 5 \text { per couple. } \\
& \rightsquigarrow \forall x\left[\text { tickets }(x) \rightarrow \mu_{\text {\#couple }_{\text {Poss }}}(x)=\frac{5 * \$}{\text { unit(\#couple })}\right] \\
& \text { Tickets for } 3 \text { couples cost } \$ 15 . \\
& \rightsquigarrow \forall x\left[\left[\text { tickets }(x) \wedge \mu_{\# \text { couple }}\right.\right. \text { Poss } \\
&
\end{aligned}
$$

The conclusion still follows from the premise, but we have fixed up the dimension denoted by couple so that it can be applied to a collection of tickets. What we have learned from this exercise is that if for every $P$, there is another dimension 'number of $P$ ', representing the number of instances of $P$ that the individual in question comprises, then there must also be 'number of $P_{R}$ ' dimensions, where $R$ can be the relation of possession; who knows what other relations can instantiate this variable.

Let us take a step back. So far, we have been pursuing the option that for every $P$, there is a dimension 'number of $P$ '. That worked for situps a day, but now $\mathscr{B}$ is infinite; we now have an infinite basis for the space of dimensions, whereas we had originally assumed it was finite. New foundations will have to be laid if we pursue this route, and work will have to be done to specify what is in the set of dimensions. Challenge Problem 3 has shown that it is more than just the set of properties (already an infinite set) that must be included; properties indexed by relations must be too.

Another option is to suppose that there is only one count dimension. This has a precedent in quantity calculus; remember, in this tradition, it is said that all numbers of things are 'dimensionless' quantities. This keeps $\mathscr{B}$ finite. But then the question becomes how to distinguish tickets per couple vs. tickets per person. So there is work to do on both horns of this dilemma.

\section{Conclusion}

I hope to have convinced the reader that it is useful to appeal to a notion of quotient among quantities across different dimensions, that it is not something that is provided for under current foundations for degree semantics, and that we can get it by importing a system of quantity calculus. I've illustrated how to do this, using the dimension-centric approach of Raposo $(2018,2019)$. At the minimum, we got a lexical entry for per out of it. But I am optimistic that much more can be built on these foundations. 
Challenge problems for a theory of degree multiplication

\section{References}

Ahn, Dorothy. 2012. Reverse quantification in Korean. Term paper, Harvard University.

Ahn, Dorothy \& Uli Sauerland. 2015. The grammar of relative measurement. In Semantics and Linguistic Theory (SALT) 25, 125-142. doi:10.3765/salt.v25i0.3062.

Ahn, Dorothy \& Uli Sauerland. 2017. Measure constructions with relative measures: Towards a syntax of non-conservative construals. The Linguistic Review doi:10.1515/tlr-2017-0001.

Alrenga, Peter. 2007. Dimensions in the semantics of comparatives: University of California at Santa Cruz $\mathrm{PhD}$ dissertation.

Anderson, Curt \& Marcin Morzycki. 2015. Degrees as kinds. Natural Language and Linguistic Theory 33. 791-828. doi:10.1007/s11049-015-9290-z.

Bale, Alan \& Bernhard Schwarz. 2019. Reverse proportionality without context dependent standards. In M. Teresa Espinal et al. (ed.), Sinn und Bedeutung 23, 93-107. Universitat Autònoma de Barcelona.

de Boer, J. 1994. On the history of quantity calculus and the international system. Metrologia 31. 405-429. doi:10.1088/0026-1394/31/6/001.

Champollion, Lucas. 2015. Stratified reference: the common core of distributivity, aspect, and measurement. Theoretical Linguistics 41(3-4). 109-149. doi:10.1515/tl-2015-0008.

Champollion, Lucas. 2016a. Covert distributivity in algebraic event semantics. Semantics \& Pragmatics 9(15). 1-65. doi:10.3765/sp.9.15.

Champollion, Lucas. 2016b. Overt distributivity in algebraic event semantics. Semantics \& Pragmatics 9(16). 1-65. doi:10.3765/sp.9.16.

Champollion, Lucas. 2017. Parts of a whole: Distributivity as a bridge between aspect and measurement. Oxford University Press. doi:10.1093/oso/9780198755128.001.0001.

Coppock, Elizabeth. under review. Part-introducing percent in English. Glossa.

Cresswell, Max J. 1977a. The semantics of degree. In Barbara Partee (ed.), Montague Grammar, 261-292. New York: Academic Press.

Cresswell, Maxwell. 1977b. Adverbs of space and time. In F. Guenthner \& S.J. Schmidt (eds.), Formal semantics and pragmatics for natural languages, 171199. Dordrecht: Reidel (reprinted in Cresswell 1985).

Cresswell, Maxwell. 1985. Adverbial modification. Dordrecht: Reidel. doi:10.1007/978-94-009-5414-4.

Dagan, Ido, Oren Glickman \& Bernardo Magnini. 2006. The PASCAL Recognising Textual Entailment challenge. In Joaquin Quiñonero-Candela, Ido Dagan, Bernardo Magnini \& Florence d'Alché Buc (eds.), Machine learning challenges: Evaluating Predictive Uncertainty, Visual Object Classification, and Recognising 
Textual Entailment, 177-190. Berlin, Heidelberg: Springer Berlin Heidelberg. doi:10.1007/11736790_9.

Ernst, Thomas. 2004. The syntax of adjuncts. Cambridge: Cambridge University Press. doi:10.1017/CBO9780511486258.

Fillmore, Charles J. 1997. Lectures on deixis. Stanford: CSLI Publications.

Heim, Irene \& Angelika Kratzer. 1998. Semantics in generative grammar. Oxford: Blackwell.

Ionin, Tania \& Ora Matushansky. 2006. The composition of complex cardinals. Journal of Semantics 23(4). 315-360. doi:10.1093/jos/ffl006.

JCGM. 2012. International vocabulary of metrology - basic and general concepts and associated terms (VIM), 3rd edition. Joint Committee for Guides in Metrology (JCGM).

Klein, Ewan. 1991. Comparatives. In Arnim von Stechow \& Dieter Wunderlich (eds.), Semantik: Ein internationales Handbuch der zeitgenössischen Forschung / Semantics: An international handbook of contemporary research, 673-691. Berlin: Walter de Gruyter.

Krantz, David H., R. Duncan Luce, Patrick Suppes \& Amos Tversky. 1971. Foundations of measurement. New York: Academic Press.

Krifka, Manfred. 1989. Nominal reference, temporal constitution and quantification in event semantics. In Renate Bartsch, Johan van Benthem \& Peter van Emde Boas (eds.), Semantics and contextual expression, 75-115. Dordrecht, Netherlands: Foris.

Krifka, Manfred. 1995. The semantics and pragmatics of polarity items. Linguistic Analysis 25. 209-257.

Li, Haoze. 2018. Event-related relative measurement. In Chris Cummins Robert Truswell, Caroline Heycock, Brian Rabern \& Hannah Rohde (eds.), Sinn und Bedeutung 21,801-818. University of Konstanz.

Lonning, Jan Tore. 1987. Mass terms and quantification. Linguistics and Philosophy 10(1). 1-52. doi:10.1007/bf00603391.

Moltmann, Friederike. 2009. Degree structure as trope structure: a trope-based analysis of positive and comparative adjectives. Linguistics and Philosophy 32. 51-94. doi:10.1007/s10988-009-9054-5.

Panaitescu, Mara \& Lucia M. Tovena. 2019. Distributivity over pairs of events and entities. In M. Teresa Espinal et al (ed.), Sinn und Bedeutung 23, 225-235. Universitat Autònoma de Barcelona.

Partee, Barbara H. 1989. Many quantifiers. In Joyce Powers \& Kenneth de Jong (eds.), ESCOL 89: Eastern States Conference on Linguistics, 383-402. Deparment of Linguistics, The Ohio State University.

Pasternak, Robert. 2019. Unifying partitive and adjective-modifying percent. Snippets 37. 77-79. doi:10.7358/snip-2019-037-past. 
Challenge problems for a theory of degree multiplication

Raposo, Álvaro P. 2018. The algebraic structure of quantity calculus. Measurement Science Review 18(4). 147-157. doi:10.1515/msr-2017-0021.

Raposo, Álvaro P. 2019. The algebraic structure of quantity calculus II: Dimensional analysis and differential and integral calculus. Measurement Science Review 19(2). 70-78. doi:10.2478/msr-2019-0012.

Rawlins, Kyle. 2013. On adverbs of (space and) time. In B. Arsenijević et al. (ed.), Studies in the Composition and Decomposition of Event Predicates, 153-197. Springer. doi:10.1007/978-94-007-5983-1_7.

van Rooij, Robert. 2011. Measurement and interadjective comparisons. Journal of Semantics 28(3). 335-358. doi:10.1093/jos/ffq018.

Rothstein, Susan. 1995. Adverbial quantification over events. Natural Language Semantics 3. 1-31.

Sassoon, Galit Weidman. 2010. Measurement theory in linguistics. Synthese 174. 151-180. doi:10.1007/s11229-009-9687-5.

Sauerland, Uli. 2014. Surface non-conservativity in German. In Christopher Piñon (ed.), Empirical Issues in Syntax and Semantics 10, 125-142. http://www.cssp.cnrs.fr/eiss10/.

Sauerland, Uli \& Robert Pasternak. to appear. German measurement structures: Case-marking and non-conservativity. Accepted for publication in the Journal of Comparative Germanic Linguistics.

Scontras, Gregory. 2017. A new kind of degree. Linguistics and Philosophy 40. 165-205. doi:10.1007/s10988-016-9200-9.

Solt, Stephanie. 2009. The semantics of adjectives of quantity. New York, NY: The City University of New York PhD dissertation.

Solt, Stephanie. 2018. Proportional comparatives and relative scales. In Robert Truswell, Chris Cummins, Caroline Heycock, Brian Rabern \& Hannah Rohde (eds.), Sinn und Bedeutung 21, 1123-1140.

Spathas, Giorgios. 2019. Plural anaphoric reference and non-conservativity. Snippets 37. 95-96. doi:10.7358/snip-2019-037-spat.

Tenny, Carol. 2000. Core events and adverbial modification. In Events as grammatical objects, Stanford: CSLI Publications.

Elizabeth Coppock

Department of Linguistics

621 Commonwealth Avenue

Boston University

ecoppock@bu.edu 